\title{
Clinical spectrum of rhabdomyolysis presented to pediatric emergency department
}

\author{
Chun-Yu Chen ${ }^{1,2}$, Yan-Ren Lin' 2,3,4 Lu-Lu Zhao ${ }^{5}$, Wen-Chieh Yang ${ }^{1,2}$, Yu-Jun Chang ${ }^{6}$, Kang-Hsi Wu ${ }^{7,8}$ \\ and Han-Ping $\mathrm{Wu}^{9,10^{*}}$
}

\begin{abstract}
Background: Rhabdomyolysis is a potentially life-threatening syndrome that can develop from a variety of causes. The aim of the work is to analyze the clinical spectrum and to evaluate the prevalence of various etiologies in children, who present to the emergency department (ED) with rhabdomyolysis.

Methods: During a 6-year study period, we retrospectively analyzed the medical charts of patients, aged 18 years or younger, with a definite diagnosis of rhabdomyolysis and serum creatinine phosphokinase (CK) levels greater than $1000 \mathrm{IU} / \mathrm{L}$. We analyzed the clinical spectrum and evaluated the potential risk factors of acute renal failure (ARF).

Results: Thirty-seven patients (mean age $=10.2 \pm 5.5$ years), including 26 males and 11 females, were enrolled in the study. Two of the most common presented symptoms in these 37 patients were muscle pain and muscle weakness (83.8\% and $73 \%$, respectively). Dark urine was reported in only $5.4 \%$ of the patients. The leading cause of rhabdomyolysis in the 0- to 9-year age group was presumed infection, and the leading cause in the 10- to 18-year age group was trauma and exercise. The incidence of ARF associated with rhabdomyolysis was $8.1 \%$ and no child needed for renal replacement therapy (RRT). We did not identify any reliable predictors of ARF or need for RRT.

Conclusions: The classic triad of symptoms of rhabdomyolysis includes myalgia, weakness and dark urine are not always presented in children. The cause of rhabdomyolysis in younger age is different from that of teenager group. However, the prognosis of rhabdomyolysis was good with appropriate management.
\end{abstract}

Keywords: Rhabdomyolysis, Emergency department, Children

\section{Background}

Rhabdomyolysis is characterized by skeletal muscle breakdown with leakage of muscle contents, including electrolytes, myoglobin, and other sarcoplasmic proteins (e.g. creatine kinase, lactate dehydrogenase, alanine aminotransferase, and aspartate aminotransferase) into the circulation $[1,2]$. Its major causes in children, include infections, trauma, exertion, drugs, toxins, metabolic disorders and electrolyte disorders [2-5]. Typically, rhabdomyolysis patients present with muscle pain, weakness, and reddish-brown urine. Nevertheless, the severity of rhabdomyolysis varies from an asymptomatic increase

\footnotetext{
* Correspondence: arthur1226@gmail.com

${ }^{9}$ Department of Pediatrics, Taichung Tzuchi Hospital, the Buddhist Medical

Foundation, Taichung, Taiwan

${ }^{10}$ Department of Medicine, School of Medicine, Tzu Chi University, Hualien,

Taiwan

Full list of author information is available at the end of the article
}

in creatine phosphokinase (CK) to heavy complications, such as acute renal failure (ARF), cardiac arrhythmias, compartmental syndrome, hypovolemic shock and disseminated intravascular coagulopathy [4-10].

As we know, ARF is the most recognized complication of rhabdomyolysis. In a larger study of 191 children with a CK level greater than $1000 \mathrm{IU} / \mathrm{L}$ who were sent to the emergency department (ED), the prevalence of ARF was $5 \%$ [11]. However, after the search of the related literature, it would appear that research discussing rhabdomyolysis in children that also presented to the pediatric ED is still rare. Therefore, in this study, we analyzed the clinical spectrum and prevalence of various etiologies in children with rhabdomyolysis that presented to the pediatric ED.

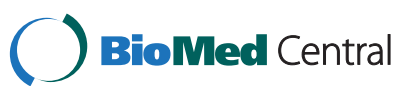

(c) 2013 Chen et al.; licensee BioMed Central Ltd. This is an Open Access article distributed under the terms of the Creative Commons Attribution License (http://creativecommons.org/licenses/by/2.0), which permits unrestricted use, distribution, and reproduction in any medium, provided the original work is properly cited. 


\section{Methods}

\section{Patient population}

This study was a single-center medical chart review of patients aged 18 years or younger who presented to the ED with a diagnosis of rhabdomyolysis based on their medical histories and elevated serum creatine kinase (CK) levels (>1000 IU/L) within 72 hours after admission to the ED. We identified potentially eligible patient visits by searching the Changhua Christian Hospital health records database. We selected charts with any of the following search terms in the primary or secondary discharge diagnosis fields: rhabdomyolysis (ICD-9 728.88), infective myositis (ICD-9 728.0), myalgia and myositis (ICD-9 729.1), and myoglobinuria (ICD-9 791.3). We reviewed 445 medical charts of all eligible patients during a 6-year period from January 2006 to December 2011. Four hundred and eight patient charts were excluded (for having initial serum CK levels < $1000 \mathrm{IU} / \mathrm{L}$ or showing a documented history of muscular dystrophy or other metabolic muscle disorder, a history of myocardial infarction, a history of chronic kidney disease, rhabdomyolysis that developed after admission to the hospital due to a coexisting condition or iatrogenic complication, or ages greater than 18 years). In total, 37 $(8.3 \%)$ of the 445 patients were included into this series. The study was approved by the Institutional Review Board of Changhua Christian Hospital.

\section{Methods}

Information about related clinical factors that was potentially causative for rhabdomyolysis were reviewed from the medical records of patients who met the inclusion criteria. The following information was obtained from the medical records of each patient: age, gender, family history, associated symptoms and signs (fever, localized muscle pain, dark urine, muscle weakness or altered mental status, muscle swelling and history of upper respiratory tract infection), laboratory tests [initial and peak serum white blood cell (WBC), hemoglobin $(\mathrm{Hb}), \mathrm{CK}$ level, electrolytes, blood urea nitrogen $(\mathrm{BUN})$, creatinine $(\mathrm{Cr})$, lactic dehydrogenase, alanine and aspartate aminotransferase levels, myoglobin levels], and urine toxicology screens for amphetamine, opiates, barbiturates, and benzodiazepines. Other clinical data included fluid administered within the first 24 hours, bicarbonate therapy, development of renal failure, need for renal replacement therapy (RRT), length of hospital stay, death, and final causes of the rhabdomyolysis and ARF. We also evaluated follow-up data, including $\mathrm{CK}, \mathrm{Cr}$, myoglobulin, and any documentation of renal sequelae.

Rhabdomyolysis was defined as serum CK greater than $1000 \mathrm{IU} / \mathrm{L}$ in the absence of any previous cardiac etiology or genetic muscular dystrophy. Acute renal failure was defined as a serum creatinine level of more than the $97.5^{\text {th }}$ percentile for the patient's age and gender [12].

Renal replacement therapy (RRT) was defined as the use of either peritoneal dialysis, hemodialysis (HD) or continuous renal replacement therapy (CRRT). We analyzed the clinical spectrum and prevalence of various etiologies in children with rhabdomyolysis presented to the ED and also evaluates the potential risk factors of ARF.

\section{Statistical analysis}

Data of categorical variables were analyzed by the chisquare test or Fisher's exact test, when appropriate. Continuous variables were analyzed by the Student's $t$-test. A $P$ value less than 0.05 was considered to be statistically significant. Distributions of variables were reported as percentages and mean \pm standard deviation (SD). Statistical analyses were performed using SPSS software (version 15.0; SPSS Inc., Chicago, IL, USA).

\section{Results}

During the 6-year study period, about 165,000 children presented to our pediatric ED. This study included 37 patients (mean age $=10.2 \pm 5.5$ years), 26 males and 11 females, who presented to the pediatric ED with rhabdomyolysis and met the inclusion criteria. Of the 37 patients, 11 (29.7\%) were younger than 7 years. Two of the most commonly presented symptoms among the 37 patients were muscle pain and muscle weakness (83.8\% and 73\%, respectively). Among them, 25 (67.6\%) presented with fever and dark urine was reported in only $5.4 \%$ of the patients (Table 1 ). Three $(8.1 \%)$ of these 37 patients were admitted to intensive care unit (ICU). The duration of hospital stay was longer in patients with ARF than those without ARF $(8.7 \pm 6.4$ days and $3.4 \pm 3.7$ days, respectively, $P=0.04$ ). All the 37

Table 1 Demographics and clinical presentations of the children with rhabdomyolysis

\begin{tabular}{|c|c|c|c|c|c|c|c|c|}
\hline \multirow[b]{2}{*}{ Variables } & & \multicolumn{2}{|c|}{$\begin{array}{c}\text { Non-ARF } \\
(n=34)\end{array}$} & \multicolumn{2}{|c|}{$\begin{array}{c}\text { ARF } \\
(n=3)\end{array}$} & \multicolumn{2}{|c|}{$\begin{array}{c}\text { Total } \\
(n=37)\end{array}$} & \multirow[b]{2}{*}{ P-value } \\
\hline & & $\mathbf{N}$ & $\%$ & $\mathbf{N}$ & $\%$ & $\mathbf{N}$ & $\%$ & \\
\hline \multirow[t]{2}{*}{ Gender } & Female & 11 & 32.4 & 0 & 0 & 11 & 29.7 & 0.540 \\
\hline & Male & 23 & 67.6 & 3 & 100 & 26 & 70.3 & \\
\hline Muscular pain & Yes & 29 & 85.3 & 2 & 66.7 & 31 & 83.8 & 0.421 \\
\hline Muscular weakness & Yes & 26 & 76.5 & 1 & 33.3 & 27 & 73 & 0.172 \\
\hline Muscular swelling & Yes & 3 & 8.8 & 0 & 0 & 3 & 8.1 & 1.000 \\
\hline Dark urine & Yes & 2 & 5.9 & 0 & 0 & 2 & 5.4 & 1.000 \\
\hline Fever & Yes & 22 & 64.7 & 3 & 100 & 25 & 67.6 & 0.537 \\
\hline \multirow[t]{3}{*}{ Admission unit } & $\mathrm{OU}$ & 14 & 41.2 & 0 & 0 & 14 & 37.8 & 0.169 \\
\hline & Ward & 18 & 52.9 & 2 & 66.7 & 20 & 54.1 & \\
\hline & $\mathrm{ICU}$ & 2 & 5.9 & 1 & 33.3 & 3 & 8.1 & \\
\hline
\end{tabular}

$A R F$ acute renal failure, $O U$ observation unit, ICU intensive care unit. 
Table 2 Etiologies of patients with rhabdomyolysis and ARF

\begin{tabular}{|c|c|c|c|c|c|c|c|}
\hline \multirow[b]{2}{*}{ Cause } & \multicolumn{2}{|c|}{$\begin{array}{l}\text { Non-ARF } \\
(n=34)\end{array}$} & \multicolumn{2}{|c|}{$\begin{array}{c}\text { ARF } \\
(n=3)\end{array}$} & \multicolumn{2}{|c|}{$\begin{array}{c}\text { Total } \\
(n=37)\end{array}$} & \multirow[b]{2}{*}{ P-value } \\
\hline & $\mathrm{N}$ & (\%) & $\mathrm{N}$ & (\%) & $\mathbf{N}$ & $(\%)$ & \\
\hline Trauma & 6 & (17.6) & 0 & $(0.0)$ & 6 & $(16.2)$ & $0.035^{*}$ \\
\hline Exercise & 6 & $(17.6)$ & 0 & $(0.0)$ & 6 & $(16.2)$ & \\
\hline Infection & 21 & $(61.8)$ & 1 & (33.3) & 22 & $(59.5)$ & \\
\hline Metabolic and electrolyte & 1 & (2.9) & 1 & (33.3) & 2 & $(5.4)$ & \\
\hline Body-temperature change & 0 & $(0.0)$ & 1 & (33.3) & 1 & $(2.7)$ & \\
\hline
\end{tabular}

*Statistically significant by the chi-square test or Fisher's exact test when appropriate.

$A R F$ acute renal failure.

patients with rhabdomyolysis had a clearly identified single diagnosis based on the patient's discharge diagnosis or diagnosis through subspecialty follow-up assessments (Table 2). The most common causes of rhabdomyolysis in this sample were infections $(\mathrm{n}=22,59.5 \%)$ and the virus was identified in 5 patients (Influenza type B in 4; Coxsackie A10 in 1). For the traumatic causes, traffic accidents $(n=6,100 \%)$ were the major cause and none of these 6 patients were complicated with ARF, including a 4-year-old girl with major trauma. For the 6 patients where involvement was caused by exercise, all of them were in the adolescent group. All cases received a large amount of fluid administration within the first 24 hours, but only one required bicarbonate therapy.

The leading cause of rhabdomyolysis in the 0 to 9 -year age group was presumed infection, and the leading cause in the 10- to 18-year age group was trauma and exercise (Figure 1). Moreover, three patients complicated with ARF and no patient needed RRT (Table 3). All 3 patients survived and had normal creatinine levels documented during follow-up visits.

The peak serum CK level in those 37 patients was $9825.1 \pm 23079.1 \mathrm{U} / \mathrm{L}$, there was no difference between the ARF and non-ARF groups (Table 4). The serum level of myoglobin was checked in half of our study patients and the peak value in those 16 patients was $1634.8 \pm$ $2334.4 \mathrm{ng} / \mathrm{mL}$. There was no significant difference between the in ARF and non-ARF groups. BUN and Creatinine were significantly higher in the ARF group compared to the non-ARF group.

\section{Discussion}

Rhabdomyolysis is a potentially life-threatening syndrome that can develop from a variety of causes; the classic triad of symptoms includes muscle pain, weakness, and dark urine and may not always be present in children [4-10,13]. In 2006, an adult study showed that $45 \%$ presented with myalgia, $38 \%$ had muscle weakness and only $3.6 \%$ noted dark urine [12]. In our study, we recorded $83.8 \%$ presented with myalgia, $73 \%$ had muscle weakness but dark urine was observed in only $5.4 \%$. There was only one patient presented with the classic triad of symptoms. In our study, we also found the male to female ratio of pediatric rhabdomyolysis was 2.3:1. The result was similar to the two other studies of rhabdomyolysis which the male to female ratio were 2:1 and $4: 1$, respectively. $[11,12]$.

The etiologies of rhabdomyolysis can be classified as hereditary and acquired. The acquired causes are further classified as traumatic and non-traumatic [14]. In pediatric patients, the most common causes are viral myositis, trauma, connective tissue disorders, seizure, physical exertion, and drug overdose [11,12]. In our series, infections accounted for more than half (59.5\%) of the causes of rhabdomyolysis, followed by trauma $(16.2 \%)$ and exercise (16.2\%). In patients that were 9 years old or younger, infections was the most common cause of rhabdomyolysis and accounted for 19 of 21 patients in this age group. However, the most common cause of rhabdomyolysis in patients older than 9 years was physical exertion. Rhabdomyolysis has been reported to be associated with a variety of viral infections, including influenza, $[15,16]$ Coxsackie virus, human immunodeficiency virus (HIV), echovirus and
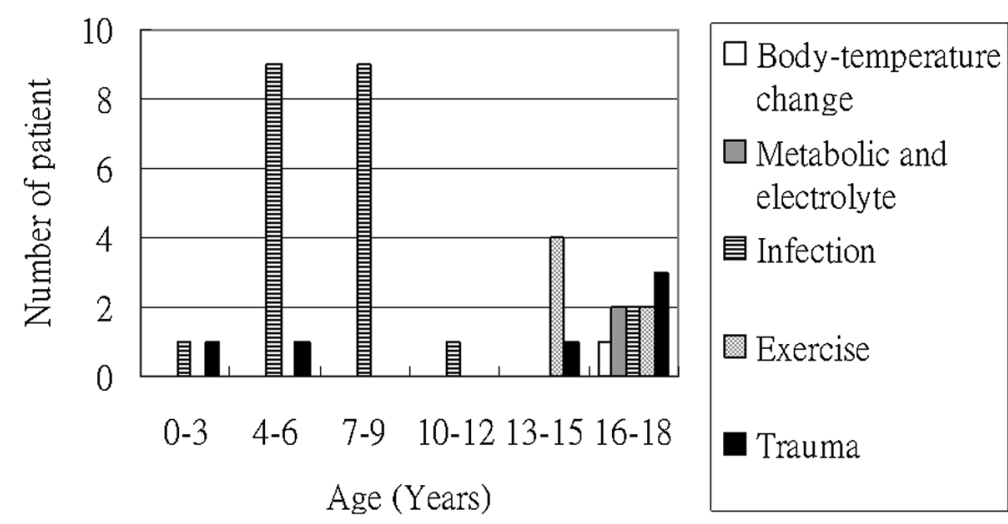

Figure 1 Etiologies of children with rhabdomyolysis in different age groups. 
Table 3 Details of the pediatric patients with acute renal failure

\begin{tabular}{|c|c|c|c|c|c|c|c|c|}
\hline Case & Presenting symptoms & Follow-up findings & $\begin{array}{c}\text { Cause of } \\
\text { rhabdomyolysis }\end{array}$ & $\begin{array}{c}\text { Initial CK } \\
\text { level (U/L) }\end{array}$ & $\begin{array}{l}\text { Peak CK } \\
\text { level (U/L) }\end{array}$ & $\begin{array}{c}\text { Peak } \\
\text { creatinine } \\
\text { level }(\mathrm{mg} / \mathrm{dl})\end{array}$ & Treatment & Outcome \\
\hline 1 & $\begin{array}{l}\text { Fever, watery diarrhea, } \\
\text { lower limbs muscle } \\
\text { pain, weakness }\end{array}$ & Septic shock & Infection & 1224 & 1224 & 3.4 & $\begin{array}{l}\text { Hydration, } \\
\text { sodium } \\
\text { bicarbonate }\end{array}$ & $\begin{array}{l}\text { No } \\
\text { dialysis, } \\
\text { alive }\end{array}$ \\
\hline 2 & $\begin{array}{c}\text { Fever, weakness, seizure } \\
\text { attack (GTC type) }\end{array}$ & $\begin{array}{l}\text { EEG: normal, brain } \\
\text { CT: minimal acute } \\
\text { subdural hematoma }\end{array}$ & Heat stroke & 1192 & 6810 & 2.2 & Hydration & $\begin{array}{l}\text { No } \\
\text { dialysis, } \\
\text { alive }\end{array}$ \\
\hline 3 & $\begin{array}{l}\text { Abdominal pain, } \\
\text { vomiting, lower limbs } \\
\text { weakness with spasm }\end{array}$ & $\begin{array}{l}\text { Congenital adrenal } \\
\text { gland hyperplasia }\end{array}$ & $\begin{array}{l}\text { Metabolic } \\
\text { disease }\end{array}$ & 1429 & 1429 & 3.8 & $\begin{array}{l}\text { Hydration, } \\
\text { furosemide }\end{array}$ & $\begin{array}{l}\text { No } \\
\text { dialysis, } \\
\text { alive }\end{array}$ \\
\hline
\end{tabular}

CK serum creatine kinase, GTC generalized tonic-clonic seizure, EEG electroencephalography, CT computed tomography.

cytomegalovirus [17]. In our series, the definite viral infection was identified in 5 patients (influenza type B in 4, Coxsackie A10 in 1) and none of them were complicated with ARF. In these 6 patients that presented with rhabdomyolysis caused by trauma, traffic accident was the major cause and none of these 6 patients were complicated with ARF.

ARF is a major life-threatening complication of rhabdomyolysis and requires immediately adequate managements. Previous studies reported the rates of ARF secondary to rhabdomyolysis range from $17-35 \%$ in adults and from $42-50 \%$ in children [18-21]. However, in the recently larger pediatric studies reported by Mannix et al [12] and Wu et al [11] it was shown that the rate of ARF with pediatric rhabdomyolysis ranged from 5-8.7 $\%$. The indications for RRT include severe and resistant hyperkalemia, an abrupt increase in potassium levels, persistent metabolic acidosis, and ongoing ARF despite conservative treatment [22-24]. Based on previous studies $[11,12]$, the rate of RRT was from $1.6-2.9 \%$ in children with rhabdomyolysis. In our study, we found a lower rate $(8.1 \%)$ of ARF, and our series showed no one treated with RRT. The outcome of patients examined in this study was considered to be good.

To prevent ARF, the early recognition and appropriate treatment of rhabdomyolysis is vital. Furthermore, the predictors of ARF have been investigated in previous studies [3,5,10-12,25]. Serum CK represents a more reliable marker than blood myoglobin for the diagnosis and assessment of the severity of rhabdomyolysis because it lasts for longer periods than blood myoglobin [3]. However, elevated serum CK has not been shown to well correlate with the severity of ARF, [5,25] although some studies showed a predictive correlation between CK and ARF $[10,11]$. Our study also indicates that the initial and peak value of CK is not a good predictor of ARF. Below are some probable reasons to explain that the peak level of CK and myoglobine is higher in the non-ARF group as compared to the ARF group in our study. First, there was a small sample size included in our study which only contained 3 patients with ARF. Second, the laboratory data were not regularly followed up in all patients, and this may lead to difficulty in determining the definite peak data. Third, the pathophysiology of rhabdomyolysis is heterogeneous and this may cause the different appearance of laboratory data based on different etiologies of rhabdomyolysis. Moreover, the definite relationship between peak CK or myoglobin and ARF caused by different etiologies of rhabdomyolysis is not clear enough. A recent adult study, conducted by Kasaoka et al [25], suggested that the serum myoglobin level on admission did not predict ARF, but that the peak value of serum myoglobin might be a predictive factor of ARF. The best cutoff value for serum myoglobin was $3865 \mathrm{ng} / \mathrm{mL}$. But due to the relatively small

Table 4 Comparison of laboratory tests of patients between AFR and non-ARF groups

\begin{tabular}{|c|c|c|c|c|c|c|c|}
\hline & \multicolumn{3}{|c|}{ Non-ARF } & \multicolumn{3}{|c|}{ ARF } & \multirow{2}{*}{$\begin{array}{c}P \text { - } \\
\text { value }\end{array}$} \\
\hline & $\mathbf{N}$ & Mean & SD & $\mathbf{N}$ & Mean & SD & \\
\hline WBC $\left(\times 10^{9} / \mathrm{L}\right)$ & 33 & 7796.1 & 5734.0 & 3 & 17663.3 & 9229.2 & 0.056 \\
\hline BUN (mg/dl) & 19 & 8.6 & 2.9 & 3 & 36.6 & 14.9 & $0.001^{*}$ \\
\hline Creatinine $(\mathrm{mg} / \mathrm{dl})$ & 31 & 0.6 & 0.3 & 3 & 3.1 & 0.8 & $<0.001^{*}$ \\
\hline ALT $(U / L)$ & 19 & 121.8 & 254.0 & 3 & 71 & 24.6 & 0.218 \\
\hline AST (U/L) & 11 & 285.1 & 354.6 & 3 & 80.0 & 40.1 & 0.555 \\
\hline Sodium (mmol/L) & 28 & 138.8 & 2.1 & 3 & 135.7 & 7.4 & 0.392 \\
\hline $\begin{array}{l}\text { Potassium (mmol/ } \\
\text { L) }\end{array}$ & 29 & 3.8 & 0.5 & 3 & 4.0 & 0.5 & 0.666 \\
\hline CK-peak (U/L) & 34 & 10413.6 & 24001.2 & 3 & 3154.3 & 3167.6 & 0.814 \\
\hline CK-initial (U/L) & 16 & 2809.2 & 3436.4 & 3 & 1281.7 & 128.6 .5 & 0.712 \\
\hline $\begin{array}{l}\text { Myoglobin-peak } \\
(\mathrm{ng} / \mathrm{mL})\end{array}$ & 15 & 1675.1 & 2410.5 & 1 & 1030 & & 0.75 \\
\hline $\begin{array}{l}\text { Myoglobin-initial } \\
(\mathrm{ng} / \mathrm{mL})\end{array}$ & 9 & 1262.2 & 1585.2 & 1 & 1030 & & 0.60 \\
\hline
\end{tabular}

*Statistically significant by Student's t-test.

$W B C$ white blood count, $B U N$ blood urea nitrogen, $A L T$ alanine

aminotransferase, AST aspartate aminotransferase, $C K$ creatine phosphokinase. 
sample size (30 patients), they could not make a definitive conclusion. However, our study showed no significant differences in laboratory findings that could help to predict ARF earlier in patients with rhabdomyolysis.

There is still a lack of randomized controlled trials for the best treatment in children with rhabdomyolysis to be conducted till now. However, regardless of underlying etiologies, the important aspects of treatments for rhabdomyolysis are considered to be conservative $[6,13,26]$. Clinically, managements may include prompt and aggressive fluid resuscitation, prevention of progressing to ARF, early correction of potentially lethal electrolyte disturbance, correction of severe metabolic acidosis, and managements of other coexisting complications $[6,13,26]$. In addition, for adults, the addition of mannitol and bicarbonate after the initial fluid resuscitation to prevent ARF has been recommended (especially for crush injury) in some studies $[13,27,28]$. But, the role of mannitol or bicarbonate in the treatment for rhabdomyolysis in children remains controversial [26]. Although causes of rhabdomyolysis are wide variety and the definite pathophysiology and development of ARF are complicated, the main therapeutic intervention to avoid ARF should be fluids and hydration $[6,13,26]$. Therefore, once the diagnosis of rhabdomyolysis in children is made in the ED, aggressive intravenous fluid rehydration will be indicated.

The present study has a number of limitations. First, in a retrospective single center review of medical records, some details of history and physical examinations may not be rigorously documented. Second, the relatively small sample size may fail to address definitely predictive factors of ARF. Finally, the laboratory data were not regularly followed up in some patients and the definite peak data was therefore difficult to record. These limitations may have led to some bias in analyzing the clinical spectrum of rhabdomyolysis in children who presented to the ED.

\section{Conclusion}

Infection was the major cause of rhabdomyolysis in children younger than 10 years; however, the leading causes in the teenager group were trauma and exercise. In our study, the classic triad of symptoms of rhabdomyolysis included myalgia, weakness and dark urine were rarely presented and the incidence of ARF associated with rhabdomyolysis was $8.1 \%$. Although our study did not identify any unique predictors of ARF or need for RRT, the prognosis of rhabdomyolysis was good with appropriate management.

\section{Competing interest}

There is no competing interest related to this study.

\section{Authors' contributions}

CYC and WCY reviewed the medical records, analyzed and interpreted the data, and drafted the manuscript; LLZ and KHW interpreted the data, and drafted the manuscript. YJC analyzed and interpreted the data. HPW designed and oversaw the study, interpreted the data, and revised the manuscript. All authors have read and approved the final manuscript for publication.

\section{Acknowledgements}

The study was partly funded by grants from the Changhua Christian Hospital (101-CCH-IRP-29) and the China Medical University Hospital (DMR-102-039).

\section{Author details}

'Division of Emergency Medicine, Department of Pediatrics, Changhua Christian Hospital, Changhua, Taiwan. ${ }^{2}$ School of Medicine, Chung Shan Medical University, Taichung, Taiwan. ${ }^{3}$ Department of Emergency Medicine, Changhua Christian Hospital, Changhua, Taiwan. ${ }^{4}$ Department of Biological Science and Technology and Institute of Biochemical Engineering, National Chiao Tung University, Hsinchu, Taiwan. ${ }^{5}$ Department of Pediatrics, Taipei Tzuchi Hospital, the Buddhist Medical Foundation, Taipei, Taiwan. ${ }^{6}$ Laboratory of Epidemiology and Biostastics, Changhua Christian Hospital, Changhua, Taiwan. ${ }^{7}$ Department of Pediatrics, China Medical University Hospital, Taichung, Taiwan. ${ }^{8}$ School of Chinese Medicine, China Medical University, Taichung, Taiwan. ${ }^{9}$ Department of Pediatrics, Taichung Tzuchi Hospital, the Buddhist Medical Foundation, Taichung, Taiwan. ${ }^{10}$ Department of Medicine, School of Medicine, Tzu Chi University, Hualien, Taiwan.

Received: 11 January 2013 Accepted: 30 August 2013 Published: 3 September 2013

\section{References}

1. Warren JD, Blumbergs PC, Thompson PD: Rhabdomyolysis: a review. Muscle Nerve 2002, 25:332-347.

2. Heyne N, Guthoff M, Weisel KC: Rhabdomyolysis and acute kidney injury. N Engl J Med 2009, 361:1412. author reply 1412-3.

3. Giannoglou GD, Chatzizisis YS, Misirli G: The syndrome of rhabdomyolysis: pathophysiology and diagnosis. Eur J Intern Med 2007, 18:90-100.

4. Allison RC, Bedsole DL: The other medical causes of rhabdomyolysis. Am J Med Sci 2003, 326:79-88.

5. Bagley WH, Yang H, Shah KH: Rhabdomyolysis. Intern Emerg Med 2007, 2:210-218.

6. Chatzizisis YS, Misirli G, Hatzitolios Al, Giannoglou GD: The syndrome of rhabdomyolysis: complications and treatment. Eur J Intern Med 2008, 19:568-574.

7. Holt S, Moore K: Pathogenesis of renal failure in rhabdomyolysis: the role of myoglobin. Exp Nephrol 2000, 8:72-76.

8. Lindner A, Zierz S: Rhabdomyolysis and myoglobinuria. Nervenarzt 2003, 74:505-515.

9. Polderman $\mathrm{KH}$ : Acute renal failure and rhabdomyolysis. Int J Artif Organs 2004, 27:1030-1033.

10. Melli G, Chaudhry V, Cornblath DR: Rhabdomyolysis: an evaluation of 475 hospitalized patients. Medicine (Baltimore) 2005, 84:377-385.

11. Wu CT, Huang JL, Lin JJ, Hsia SH: Factors associated with nontraumatic rhabdomyolysis and acute renal failure of children in Taiwan population. Pediatr Emerg Care 2009, 25:657-660.

12. Mannix $R$, Tan ML, Wright $R$, Baskin M: Acute pediatric rhabdomyolysis: causes and rates of renal failure. Pediatrics 2006, 118:2119-2125.

13. Khan FY: Rhabdomyolysis: a review of the literature. Neth J Med 2009, 67:272-283.

14. Beetham R: Biochemical investigation of suspected rhabdomyolysis. Ann Clin Biochem 2000, 37:581-587.

15. Wu CT, Hsia SH, Huang JL: Influenza B-associated rhabdomyolysis in Taiwanese children. Acta Paediatr 2010, 99:1701-1704.

16. Knochel JP: Rhabdomyolysis and myoglobinuria. Semin Nephrol 1982, $1: 75-86$.

17. Pesik NT, Otten EJ: Severe rhabdomyolysis following a viral illness: a case report and review of the literature. J Emerg Med 1996, 14:425-428.

18. Gabow PA, Kaehny WD, Kelleher SP: The spectrum of rhabdomyolysis. Medicine 1982, 61:141-152.

19. Ward MM: Factors predictive of acute renal failure in rhabdomyolysis. Arch Intern Med 1988, 148:1553-1557. 
20. Watanabe T: Rhabdomyolysis and acute renal failure in children. Pediatr Nephrol 2001, 16:1072-1075.

21. Watemberg N, Leshner RL, Armstrong BA, Lerman-Sagie T: Acute pediatric rhabdomyolysis. J Child Neurol 2000, 15:222-227.

22. Vanholder R, Sever MS, Erek E, Lameire N: Rhabdomyolysis. J Am Soc Nephrol 2000, 11:1553-1556.

23. Gill N, Nally JV Jr, Fatica RA: Renal failure secondary to acute tubular necrosis: epidemiology, diagnosis, and management. Chest 2005, 128:2847-2863.

24. Russell TA: Acute renal failure related to rhabdomyolysis: pathophysiology, diagnosis and collaborative management. Nephrol Nurs J 2000, 27:567-575.

25. Kasaoka S, Todani M, Kaneko T, Kawamura Y, Oda Y, Tsuruta R, et al: Peak value of blood myoglobin predicts acute renal failure induced by rhabdomyolysis. J Crit Care 2010, 25:601-604.

26. Elsayed EF, Reilly RF: Rhabdomyolysis: a review, with emphasis on the pediatric population. Pediatr Nephrol 2010, 25:7-18.

27. Altintepe L, Guney I, Tonbul Z, Türk S, Mazi M, Ağca E, et al: Early and intensive fluid replacement prevents acute renal failure in the crush cases associated with spontaneous collapse of an apartment in Konya. Ren Fail 2007, 29:737-741.

28. Better OS, Rubinstein I: Management of shock and acute renal failure in casualties suffering from the crush syndrome. Renal Fail 1997, 19:647-653.

doi:10.1186/1471-2431-13-134

Cite this article as: Chen et al:: Clinical spectrum of rhabdomyolysis presented to pediatric emergency department. BMC Pediatrics 2013 13:134.

\section{Submit your next manuscript to BioMed Central and take full advantage of:}

- Convenient online submission

- Thorough peer review

- No space constraints or color figure charges

- Immediate publication on acceptance

- Inclusion in PubMed, CAS, Scopus and Google Scholar

- Research which is freely available for redistribution 